\title{
Assessment of third ventriculostomy patency with the 3D-SPACE technique: a preliminary multicenter research study
}

\author{
Oktay Algin, MD, ${ }^{1,2}$ Murat Ucar, MD, ${ }^{3}$ Evrim Ozmen, MD, ${ }^{1}$ Alp Ozgun Borcek, MD, ${ }^{4}$ \\ Pinar Ozisik, MD, ${ }^{5}$ Gokhan Ocakoglu, PhD, ${ }^{6}$ and E. Turgut Tali, MD ${ }^{3}$
}

1Department of Radiology, Ataturk Training and Research Hospital; 'Bilkent University, National MR Research Center (UMRAM), Bilkent, Ankara; Departments of ${ }^{3}$ Radiology and ${ }^{4}$ Neurosurgery, Gazi University Medical Faculty; ${ }^{5}$ Neurosurgery Clinic, Koru Hospital, Ankara; and ${ }^{6}$ Biostatistics, Uludag University, Gorukle, Bursa, Turkey

OBJECT The goal of this study was to determine the value of the 3D sampling perfection with application-optimized contrasts using different flip-angle evolutions (3D-SPACE) technique in the evaluation of endoscopic third ventriculostomy (ETV) patency.

METHODS Twenty-six patients with ETV were examined using 3-T MRI units. Sagittal-plane 3D-SPACE with variant flip-angle mode, 3D T1-weighted (T1W), and 3D heavily T2-weighted (T2W) images were obtained with isotropic voxel sizes. Also, sagittal-axial plane phase-contrast cine (PC)-MR images were obtained. The following findings were evaluated: diameters of stoma and third ventricle, flow-void sign on 3D-SPACE and PC-MR images, integrity of the third ventricle on heavily $\mathrm{T} 2 \mathrm{~W}$ images, and quantitative PC-MRI parameters of the stoma. Obtained sequences were evaluated singly, in combination with one another, and all together.

RESULTS The mean area, flow, and velocity values measured at the level of stoma in patients with patent stoma were significantly higher than those measured in patients with closed stoma $(p<0.05)$. There was significant correlation among PC-MRI, 3D-SPACE, and 3D heavily T2W techniques regarding assessment of ETV patency $(p<0.001)$. The 3D-SPACE technique provided the lowest rate of ambiguous results.

CONCLUSIONS The 3D-SPACE technique seems to be the most efficient one for determination of ETV patency. The authors suggest the use of 3D-SPACE as a stand-alone first-line sequence in addition to routine brain MRI protocols in assessing patients with ETV, thereby decreasing scan time and reserving the use of a combination of additional sequences such as PC-MRI and 3D heavily T2W images in suspicious or complex cases.

http://thejns.org/doi/abs/10.3171/2014.10.JNS14298

KEY WORDS magnetic resonance imaging; obstructive hydrocephalus; cerebrospinal fluid; 3D sampling perfection with application-optimized contrasts using different flip-angle evolutions; endoscopic third ventriculostomy; ventriculoperitoneal shunt

$\mathrm{E}$ NDOSCOPIC third ventriculostomy (ETV) is a minimally invasive, safe, and relatively new CSF diversion method; it is mostly used in aqueductal stenosis, but may also be indicated in the treatment of other causes of hydrocephalus. ${ }^{1}$ With this procedure the inferior wall of the third ventricle is fenestrated, and CSF in the ventricular system is diverted into the prepontine cisternthereby bypassing the obstruction - and CSF circulation is reestablished..$^{4,7}$ It is a more physiological treatment option compared with shunt procedures, and therefore it is being done with increasing frequency. $4,7,10$

Clinical, laboratory, and conventional imaging findings may be useful to determine whether the ETV stoma is patent, although advanced radiological methods are mostly required. 2,6 Ventriculography is a gold-standard technique for determination of ETV patency. ${ }^{4}$ On the other hand,

ABBREVIATIONS ETV = endoscopic third ventriculostomy; PC = phase-contrast cine; T1W, T2W = T1-, T2-weighted; 3D-SPACE = 3D sampling perfection with application-optimized contrasts using different flip-angle evolutions.

SUBMITTED March 10, 2014. ACCEPTED October 15, 2014.

INCLUDE WHEN CITING Published online April 10, 2015; DOI: 10.3171/2014.10.JNS14298.

DISCLOSURE The authors report no conflict of interest concerning the materials or methods used in this study or the findings specified in this paper. 
routine use of ventriculography is completely obsolete and contraindicated because it is extraordinarily invasive and can be replaced by noninvasive procedures such as sagittal-plane T1- and T2-weighted (T1W, T2W) sequences with thin slice-thickness, 3D heavily T2W sequences (e.g., 3D constructive interference in steady state [3D-CISS]), and/or phase-contrast cine (PC) MRI. ${ }^{6-8}$ However, each of these techniques has its own limitations and drawbacks. ${ }^{2,5}$

With the development of MRI technology, the whole cranium, including CSF-containing spaces (especially the ventricular system), and CSF circulation are better evaluated with 3D isotropic submillimetric data obtained by 3-T MR units.,11 By using a relatively new technique called 3D sampling perfection with application-optimized contrasts using different flip-angle evolutions (3D-SPACE) developed by Mugler et al., it has now become possible to scan the whole cranium with $<1-\mathrm{mm}^{3}$ voxels within an acceptable time period and without exceeding specific absorption rate limits in 3-T scanners. ${ }^{14,15}$ The 3D-SPACE technique is an optimized, single-slab, turbo spin echobased method in which variable flip angles are used, and the adoption of this method in 3-T scanners is increasing in frequency. ${ }^{12}$

In our recently published studies we have concluded that the 3D-SPACE technique provides useful information in the determination of type and cause of hydrocephalus, integrity of the third ventricle floor, location and severity of obstruction, and demonstration of CSF circulation. ${ }^{3,5}$ The primary purpose of this preliminary study is to determine the value of the 3D-SPACE technique in the evaluation of ETV patency. Our secondary aim is to compare 3D-SPACE with other MR sequences for the diagnosis of ETV patency. To the best of our knowledge, there is no study reporting on the value of this technique in the evaluation of ETV.

\section{Methods \\ Study Population}

All participants gave their informed consent to participate in this institutional review board-approved 2-center retrospective study. All patients treated with ETV between 2010 and 2013 were included in the study. Exclusion criteria were as follows: 1) contraindications for MRI [such as having a pacemaker (1 patient), metallic implants (3 patients), or claustrophobia (1 patient)]; and 2) significant motion artifacts ( 2 patients). In total, 7 patients were excluded due to these criteria. The final number of patients was 26 (12 female and 14 male patients; mean age 22 years; range 2-74 years). The reasons for the MR examinations are routine follow-up or specific complaints of the patients (such as headache, visual disturbance, and/or syncope).

\section{The MRI Protocol and Evaluation of the Images}

All MRI examinations were performed in 2 universities by 2 different 3-T MR scanners (Trio and Verio units, Siemens Medical Solutions). All patients underwent scanning in the supine position by using a birdcage multichannel head-array coil and parallel imaging techniques. After acquisition of scout images, sagittal-plane 3D-T1W, 3D-
SPACE with variant flip-angle mode, and 3D heavily T2W images were obtained with isotropic voxel sizes. Also, axial and midline sagittal-plane 2D PC-MRI studies with retrospective cardiac gating were obtained. In each PCMRI acquisition, 24 magnitude and phase images were obtained. The imaging time of each PC-MRI acquisition varied according to the patients' heart rates. Total MRI time was approximately 30 minutes for each patient. The data regarding the details of MRI protocols are given in Table 1. All MRI examinations were performed by 2 experienced technicians.

All images obtained in the 2 centers were evaluated by 2 experienced neuroradiologists (O.A., M.U.) on 2 different workstations with the same configuration and menu. All reformatted images from 3D sequences were also available. For the assessment of ETV patency, the following findings were evaluated using the Argus flow analysis program and 3D tools of the dedicated workstations (Leonardo, Siemens): Evans index; diameters of stoma and the third ventricle; presence of flow-void sign on 3DSPACE and PC-MR images; integrity of the third ventricle on 3D heavily T2W images; and quantitative PC-MRI parameters of the stoma.

The third ventricle diameter was determined by measuring the widest transverse distance on coronal reformatted 3D-SPACE images. Diameter of the ETV stoma was measured on both sagittal and coronal reformatted 3D-SPACE images, and the mean value of these 2 measurements was accepted as the diameter of the stoma. Quantitative PC-MRI parameters of the stoma were obtained on axial PC-MRI scans passing through the level of stoma; this was done using Argus software to place a circular region of interest on the stoma that would not exceed its borders. Evans index was calculated in the same way as demonstrated in the previous studies (the ratio of the greatest distance between the outer borders of the frontal horns to the greatest distance between the inner borders of the inner tabulae on the same slice is called the Evans index). ${ }^{13}$ The presence of flow-void sign in the ETV stoma on 3D-SPACE and PC-MRI studies and integrity of the third ventricle on 3D heavily T2W images were scored on 3 -point scales according to the following scoring schema.

\section{Flow-Void Scoring of SPACE Sequence}

Score 0 (closed or nonfunctional stoma): no flow was detected at the level of the stoma. The CSF at the level of the third ventricle floor showed hyperintense signal. Additionally, intact floor of the third ventricle was clearly visible. Score 1 (patent and functional ETV): flow-void sign passing from the third ventricle through the prepontine cistern across the stoma was clearly visible. Score 2 (suspected findings): the floor of the third ventricle could not be seen clearly, and/or flow-void sign was minimally visible at the level of the stoma.

\section{Flow-Void Scoring of PC-MR Images}

Score 0 (closed or nonfunctional stoma): there was no pulsatile CSF flow across the stoma. Score 1 (patent and functional ETV): CSF flow with flush-fill periods between the third ventricle floor and prepontine cistern could be 
TABLE 1. Sequences and parameters of the 3-T MRI examinations used in 26 patients with ETV

\begin{tabular}{|c|c|c|c|c|c|}
\hline MRI Unit \& Parameter & 3D-MPRAGE & 3D-SPACE & PC-MRI (qualitative) & 3D Heavily $T 2 W^{*}$ & PC-MRI (quantitative) \\
\hline \multicolumn{6}{|l|}{ Trio } \\
\hline TR/TE (msec) & $2130 / 3.45$ & $3000 / 579$ & $34.9 / 9.8$ & $3000 / 526$ & $30 / 7.43$ \\
\hline TI (msec) & 1100 & NA & NA & NA & NA \\
\hline Slice thickness (mm) & 0.8 & 0.6 & 4 & 0.7 & 4 \\
\hline $\mathrm{FOV}(\mathrm{mm})$ & $230 \times 230$ & $240 \times 240$ & $240 \times 240$ & $240 \times 240$ & $240 \times 240$ \\
\hline Acquisition time (min) & 5.5 & 6 & 5 & 5 & 5 \\
\hline Velocity encoding $(\mathrm{cm} / \mathrm{sec})$ & NA & NA & 6 & NA & 20 \\
\hline NEX & 1 & 2 & 2 & 2 & 1 \\
\hline No. of slices & 240 & 240 & 1 & 240 & 1 \\
\hline FA & $8^{\circ}$ & Variable & $10^{\circ}$ & $120^{\circ}$ & $10^{\circ}$ \\
\hline Imaging plane & Sagittal & Sagittal & Axial-sagittal & Sagittal & Axial \\
\hline Distance factor & $50 \%$ & NA & NA & NA & NA \\
\hline PAT factor & 2 & 2 & None & 2 & None \\
\hline PAT mode & GRAPPA & GRAPPA & NA & GRAPPA & NA \\
\hline Voxel size (mm) & $0.8 \times 0.8 \times 0.8$ & $0.6 \times 0.6 \times 0.6$ & NA & $0.7 \times 0.7 \times 0.7$ & NA \\
\hline FA mode & NA & T2 variant & NA & T2 constant & NA \\
\hline \multicolumn{6}{|l|}{ Verio } \\
\hline TR/TE (msec) & $1900 / 2.52$ & $3200 / 411$ & $36 / 5.6$ & $8.4 / 3.8$ & $34.5 / 5.8$ \\
\hline $\mathrm{TI}$ (msec) & 900 & NA & NA & NA & NA \\
\hline Slice thickness (mm) & 1 & 0.8 & 4 & 0.6 & 4 \\
\hline $\mathrm{FOV}(\mathrm{mm})$ & $250 \times 250$ & $210 \times 210$ & $240 \times 240$ & $180 \times 180$ & $240 \times 240$ \\
\hline Acquisition time (min) & 4.5 & 5 & 4-5 & 5 & 5 \\
\hline Velocity encoding $(\mathrm{cm} / \mathrm{sec})$ & NA & NA & $6-8$ & NA & $20-22$ \\
\hline NEX & 1 & 2 & 1 & 2 & 1 \\
\hline No. of slices & 240 & 240 & 1 & 240 & 1 \\
\hline FA & $9^{\circ}$ & Variable & $20^{\circ}$ & $50^{\circ}$ & $20^{\circ}$ \\
\hline Imaging plane & Sagittal & Sagittal & Axial-sagittal & Sagittal & Axial \\
\hline Distance factor & $50 \%$ & NA & NA & NA & NA \\
\hline PAT factor & 2 & 2 & None & 2 & None \\
\hline PAT mode & GRAPPA & GRAPPA & NA & GRAPPA & NA \\
\hline Voxel size $(\mathrm{mm})$ & $1 \times 1 \times 1$ & $0.8 \times 0.8 \times 0.8$ & NA & $0.6 \times 0.6 \times 0.6$ & NA \\
\hline FA mode & NA & T2 variant & NA & NA & NA \\
\hline
\end{tabular}

FA = flip angle; FOV = field of view; GRAPPA = generalized auto-calibrating partially parallel acquisitions; MPRAGE = T1W magnetization-prepared rapid acquisition gradient echo; NA = not applicable; NEX = number of excitations; $P A T$ = parallel acquisition technique.

* For the Trio unit, the 3D heavily T2W sequences were acquired using the 3D-SPACE technique, and for the Verio unit, the 3D constructive interference in steady state (3D-CISS) technique was used.

clearly seen throughout the whole cardiac cycle. Score 2 (suspected findings): minimal flow was present across the stoma, but could not be seen on all phase images.

\section{Scoring of Third Ventricle Integrity on 3D Heavily T2W Images}

Score 0 (closed stoma): there were no defects in the ventricle floor. Score 1 (patent ETV): the stoma was clearly visible as a prominent defect. Score 2 (suspected findings): the inferior wall of the third ventricle could not be completely visualized, or the presence of the defect was equivocal.

In this study group, scoring for each sequence was first done independently from the other 2 sequences, by the 2 readers in consensus. Hence, a total of 3 scoring sessions were held initially, 1 week apart from each other for each sequence (a session is defined as 1 review of the entire group of study patients from the first to the last).

Then, 3 more scoring sessions were undertaken by the readers, this time using double-sequence combinations in every single patient (i.e., 3D-SPACE and 3D heavily T2W combined; 3D-SPACE and PC-MRI combined; and 3D heavily T2W and PC-MRI combined). Each session was titled according to whatever "sequence combination" was used, and the sessions were also 1 week apart from each other.

Finally, a triple combination session was held, in which all of the 3 (i.e., 3D heavily T2W, 3D-SPACE, and PCMRI) sequences were taken together into consideration for making a scoring decision on every single patient in 
the study. For the same reason we put 1-week intervals between the scoring sessions (i.e., to avoid bias that may originate from memory effects), none of the scoring sessions had a similar patient order while evaluations were done. Final decisions on scoring and other imaging findings in the patients were given by consensus between the 2 neuroradiologists, who were blinded to clinical and surgical data of the patients during scoring.

After all of the above-mentioned scoring sessions were finalized, the results obtained from 3D-SPACE, 3D heavily T2W, and PC-MRI sequences were statistically compared with the gold-standard reference, which was set for this study as an available combination of at least 2 of the following 4 criteria: 1) clinical findings; 2) endoscopic findings; 3) long-term clinical follow-up (> 20 months); and/or 4) long-term radiological follow-up (2 years).

\section{Statistical Analysis}

Statistical analysis was performed using SPSS version 20.0 (IBM, Inc.). Continuous variables were expressed as the median (minimum, maximum) whereas categorical variables were expressed by frequency and related percentage values. Continuous variable between-group comparisons were performed using the Mann-Whitney test. Chisquare and Fisher's exact tests were performed to compare categorical variables between the groups. Agreement results of reference tests against PC-MRI, 3D heavily T2W, and 3D-SPACE findings for the evaluation of ETV patency were examined using the kappa coefficient. Data from the patients categorized as Score 2 (suspected findings) were not included in the comparison analysis. The $\mathrm{p}$ value was set at $\leq 0.05$ for statistical significance.

\section{Results}

Aqueductal stenosis was detected in 21 patients in the study group, complex hydrocephalus (with meningitis history) in 2 of them, fourth ventricular outlet obstruction in 2 of them (Chiari malformation in one patient and medulloblastoma in the other), and in 1 of them a cyst in the cavum septum pellucidum was detected. Among the $21 \mathrm{pa}-$ tients with aqueductal stenosis, obstruction was secondary to aqueductal web formation in 7 , tectal glioma in 6 , and arachnoid cyst in the quadrigeminal cistern in 2 (Fig. 1). Nonfunctional or closed stoma was detected in 7 patients. In the other 19 patients, a functional and patent ETV was present (Tables 2 and 3).

The Evans index was above 0.3 for all patients; the mean Evans index was calculated as 0.44 (range 0.320.76). Evans indexes calculated for those patients with patent stoma were significantly lower than those with closed or nonfunctional stoma $(\mathrm{p}=0.023)$. There were no significant differences according to sex and age between the groups $(\mathrm{p}>0.05)$. The mean third ventricle and stoma diameters were $16 \mathrm{~mm}$ (range 9-23 $\mathrm{mm}$ ) and $3 \mathrm{~mm}$ (range $1.2-5.8 \mathrm{~mm}$ ), respectively. Area, mean flow, and velocity measurements taken at the level of the stoma in patients with patent ETV were significantly higher than those ob-
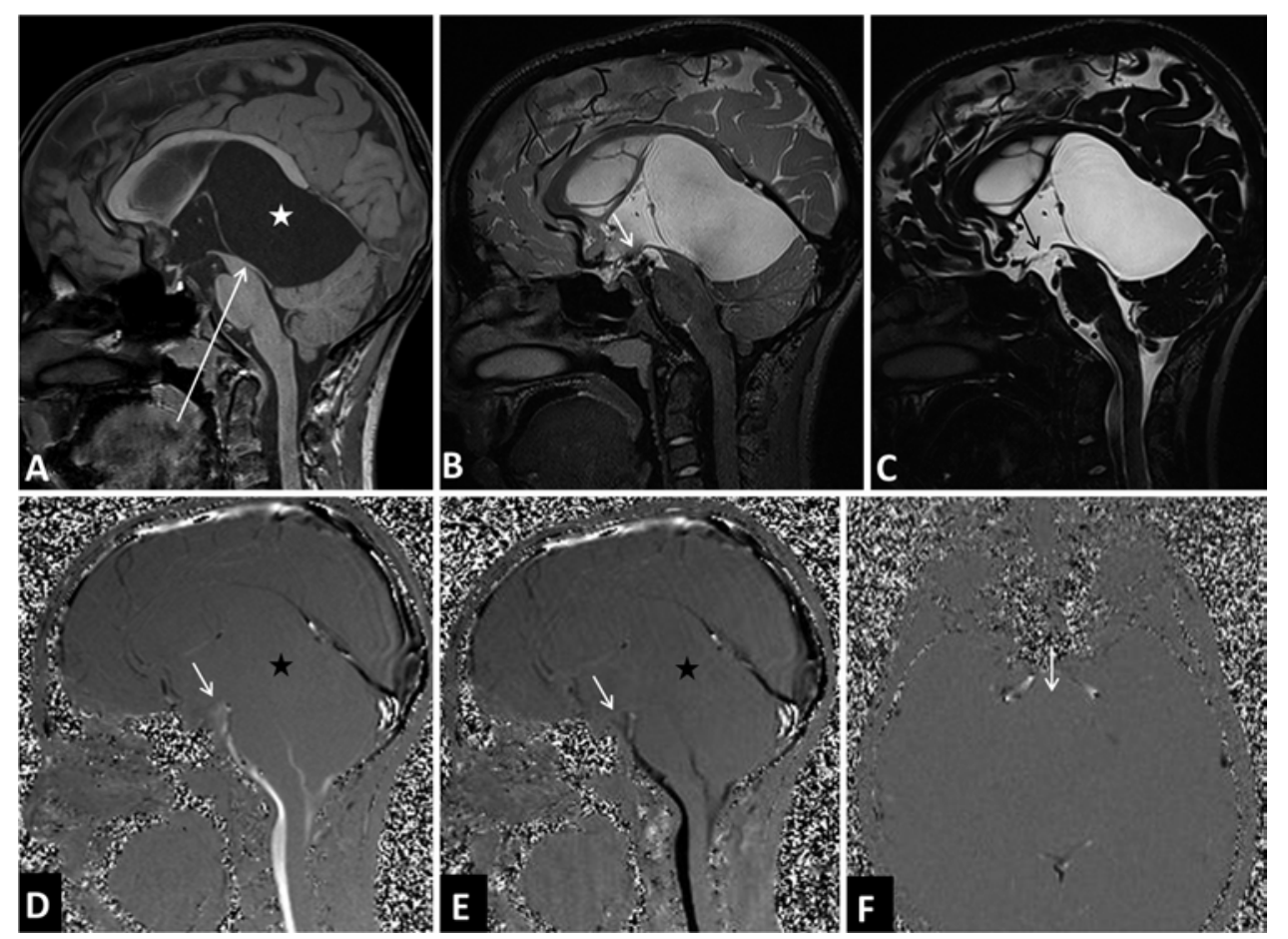

FIG. 1. Sagittal 3D-MPRAGE image of a 10-year-old boy shows arachnoid cyst (star) localized in the quadrigeminal cistern and aqueductal stenosis due to compression (arrow in A). Sagittal 3D-SPACE image shows neither flow voids nor any other signs of flow at the level of ETV stoma (arrow in B). In a sagittal 3D heavily T2W image a defect is present in the inferior wall of the third ventricle, but it is shown that the stoma is narrow (arrow in C). It is not possible to assess the ETV patency by using only 3D heavily T2W images. On sagittal and axial PC-MR images (D-F), no flow is shown at the level of the stoma (arrows), and the arachnoid cyst in the quadrigeminal cistern is detected to be a noncommunicating type (stars). 
TABLE 2. Analysis of the study variables in nonfunctional and patent ETV groups*

\begin{tabular}{llll}
\hline \multicolumn{1}{c}{ Variable } & Nonfunctional & Patent & $p$ Value \\
\hline Age in yrs & $8(2,37)$ & $25(4,74)$ & 0.083 \\
\hline Third ventricle diameter in $\mathrm{mm}$ & $16.5(10,22)$ & $15(9,23)$ & 0.929 \\
\hline Stoma diameter in $\mathrm{mm}$ & $3(2,4)$ & $3(1.2,5.8)$ & ND \\
\hline Evans index & $0.55(0.38,0.76)$ & $0.39(0.32,0.60)$ & 0.023 \\
\hline Net forward vol in $\mathrm{ml}$ & $0(0,0.002)$ & $0.03(-0.04,0.51)$ & 0.015 \\
\hline Peak velocity in cm/sec & $0(0,5.88)$ & $5.37(-5.55,17.14)$ & 0.108 \\
\hline Average velocity in cm/sec & $0(0,0.10)$ & $0.46(-1.11,1.82)$ & 0.028 \\
\hline Area in cm ${ }^{2}$ & $0.02(0,0.11)$ & $0.12(0.03,0.40)$ & 0.001 \\
\hline Average flow over range in $\mathrm{ml} / \mathrm{sec}$ & $0(0,0.002)$ & $0.06(-0.15,0.73)$ & 0.036 \\
\hline Forward vol in $\mathrm{ml}$ & $0(0,0.05)$ & $0.05(-0.03,0.51)$ & 0.004 \\
\hline Reverse vol in $\mathrm{ml}$ & $0(0,0.05)$ & $0.05(0.01,0.16)$ & 0.002 \\
\hline
\end{tabular}

$\mathrm{ND}=$ not done

* Except as otherwise stated, values are expressed as the median (minimum, maximum).

$\dagger$ Statistical analysis could not be performed due to insufficient sample size.

TABLE 3. Patency scores of ETV assessed using 3D heavily T2W, PC-MRI, and 3D-SPACE sequences in 26 patients*

\begin{tabular}{|c|c|c|c|c|c|c|c|c|c|}
\hline $\begin{array}{l}\text { Case } \\
\text { No. }\end{array}$ & $\begin{array}{l}\text { Age (yrs), } \\
\text { Sex }\end{array}$ & $\begin{array}{l}\text { 3D Heavily } \\
\text { T2W \& } \\
\text { PC-MRI }\end{array}$ & $\begin{array}{l}\text { 3D Heavily } \\
\text { T2W \& } \\
\text { 3D-SPACE }\end{array}$ & $\begin{array}{l}\text { 3D-SPACE } \\
\text { \& PC-MRI }\end{array}$ & $\begin{array}{l}\text { 3D Heavily T2W } \\
\text { \& PC-MRI \& } \\
\text { 3D-SPACE }\end{array}$ & $\begin{array}{c}\text { 3D Heavily } \\
\text { T2W }\end{array}$ & 3D-SPACE & PC-MRI & $\begin{array}{l}\text { GS Test } \\
\text { Results }\end{array}$ \\
\hline 1 & $4, \mathrm{~F}$ & 0 & 0 & 0 & 0 & 0 & 0 & 0 & 0 \\
\hline 2 & $41, \mathrm{M}$ & 1 & 1 & 1 & 1 & 1 & 1 & 1 & 1 \\
\hline 3 & $8, F$ & 0 & 0 & 0 & 0 & 0 & 0 & 0 & 0 \\
\hline 4 & $9, M$ & 2 & 1 & 1 & 1 & 1 & 1 & 2 & 1 \\
\hline 5 & $7, \mathrm{M}$ & 1 & 1 & 1 & 1 & 1 & 1 & 1 & 1 \\
\hline 6 & $16, \mathrm{M}$ & 1 & 1 & 1 & 0 & 1 & 1 & 1 & 0 \\
\hline 7 & $8, M$ & 0 & 0 & 0 & 0 & 2 & 0 & 0 & 0 \\
\hline 8 & $4, M$ & 1 & 1 & 1 & 1 & 1 & 1 & 1 & 1 \\
\hline 9 & $74, \mathrm{~F}$ & 1 & 1 & 1 & 1 & 1 & 1 & 1 & 1 \\
\hline 10 & $10, M$ & 0 & 0 & 0 & 0 & 0 & 0 & 0 & 0 \\
\hline 11 & $39, \mathrm{M}$ & 1 & 1 & 1 & 1 & 1 & 1 & 1 & 1 \\
\hline 12 & $5, F$ & 1 & 1 & 1 & 1 & 1 & 1 & 1 & 1 \\
\hline 13 & $33, \mathrm{~F}$ & 1 & 1 & 1 & 1 & 1 & 1 & 1 & 1 \\
\hline 14 & $2, \mathrm{~F}$ & 2 & 2 & 2 & 0 & 2 & 0 & 0 & 0 \\
\hline 15 & $29, F$ & 1 & 1 & 1 & 1 & 1 & 1 & 1 & 1 \\
\hline 16 & $22, \mathrm{M}$ & 1 & 1 & 1 & 1 & 1 & 1 & 1 & 1 \\
\hline 17 & $27, \mathrm{M}$ & 1 & 1 & 1 & 1 & 1 & 1 & 1 & 1 \\
\hline 18 & $8, M$ & 1 & 1 & 1 & 1 & 1 & 1 & 1 & 1 \\
\hline 19 & $14, \mathrm{M}$ & 1 & 1 & 1 & 1 & 1 & 1 & 1 & 1 \\
\hline 20 & $29, \mathrm{M}$ & 1 & 1 & 1 & 1 & 2 & 1 & 1 & 1 \\
\hline 21 & $31, \mathrm{~F}$ & 1 & 1 & 1 & 1 & 1 & 1 & 1 & 1 \\
\hline 22 & $37, \mathrm{~F}$ & 1 & 1 & 1 & 1 & 1 & 1 & 1 & 1 \\
\hline 23 & $16, F$ & 1 & 1 & 1 & 1 & 1 & 1 & 1 & 1 \\
\hline 24 & $23, \mathrm{~F}$ & 2 & 1 & 1 & 1 & 2 & 1 & 1 & 1 \\
\hline 25 & $37, \mathrm{~F}$ & 0 & 0 & 0 & 0 & 0 & 0 & 0 & 0 \\
\hline 26 & $29, \mathrm{M}$ & 1 & 1 & 1 & 1 & 1 & 1 & 1 & 1 \\
\hline
\end{tabular}

$\mathrm{GS}=$ gold standard.

* Score 0, closed or nonfunctional stoma; Score 1, patent ETV; Score 2, suspected findings. 

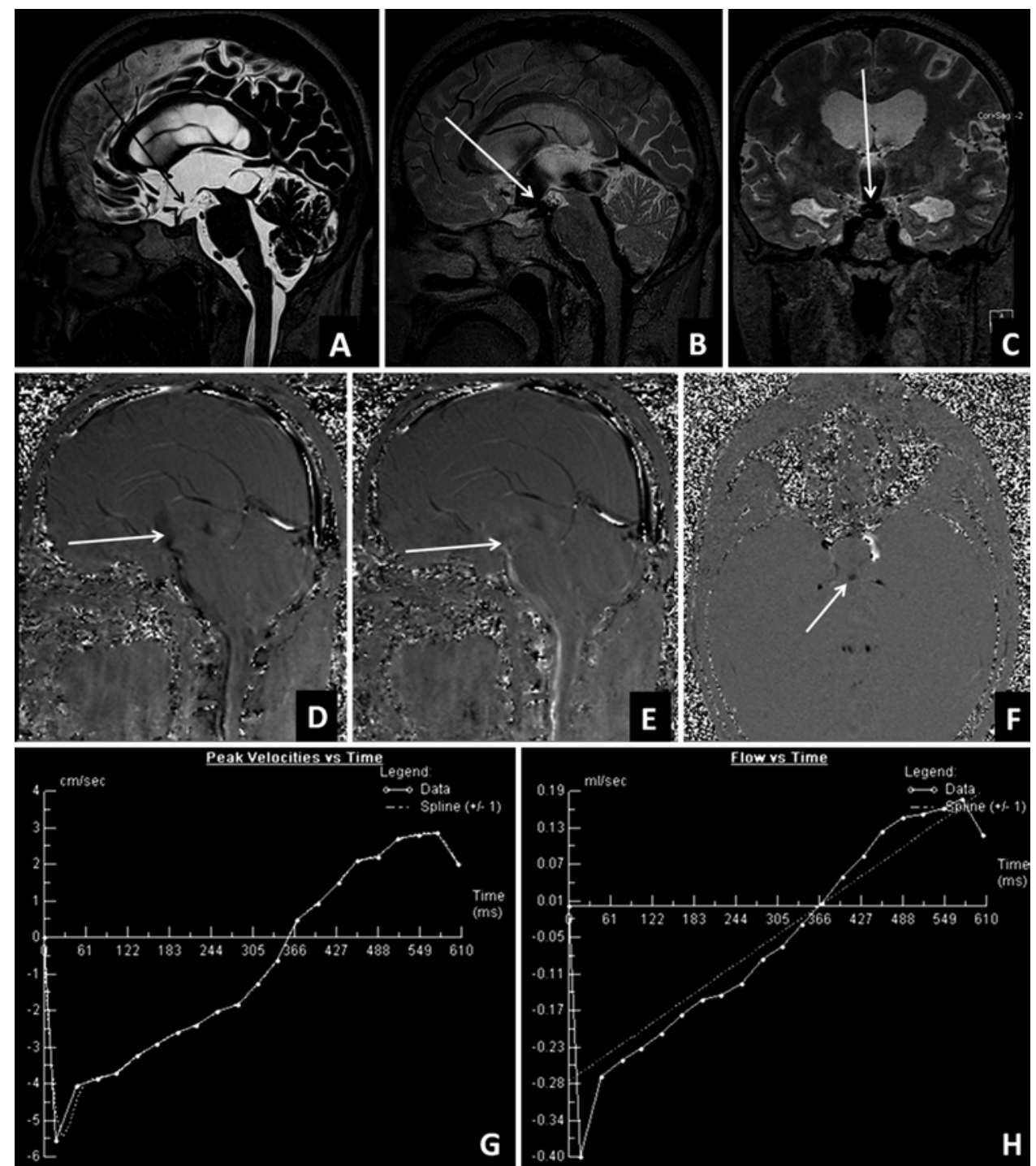

FIG. 2. A 33-year-old woman with aqueductal stenosis secondary to web formation. Sagittal 3D heavily T2W image demonstrates clearly that the stoma is patent (arrow in A). On sagittal and reformatted coronal 3D-SPACE images, the flow-void phenomenon (that is, hypointense flow void starting from the anterior part of the third ventricle and reaching to the prepontine cistern), consistent with a functional stoma, is shown (arrows in B and C). The CSF flow through the prepontine cistern toward the third ventricle is shown on sagittal and axial PC-MR images, consistent with a patent stoma (arrows, D-F). Quantitative CSF flow analysis on PCMR images across the stoma reveals that peak velocity is $-5.55 \mathrm{~cm} / \mathrm{sec}(\mathbf{G})$, and stroke volume is $-0.033 \mathrm{ml} / \mathrm{sec}(\mathrm{H})$.

tained in patients with closed or nonfunctional ETV $(\mathrm{p}<$ 0.05 , Table 2, Fig. 2).

In 20 patients $(77 \%)$, results obtained from each of the PC-MRI, 3D-SPACE, and 3D heavily T2W sequences were highly correlated with those obtained from goldstandard criteria, as defined at the end of the Methods section (Fig. 3). In 4 of the remaining 6 patients the 3D heavily T2W findings, and in 1 of them the PC-MRI findings were equivocal (Score 2). However, in these 5 patients the findings obtained from the other sequences showed a high correlation with those obtained from the aforementioned gold-standard criteria. In 1 patient, although no accurate diagnosis was achieved when PC-MRI, 3D-SPACE, and/ or 3D heavily $\mathrm{T} 2 \mathrm{~W}$ images were evaluated individually, an accurate diagnosis was achieved when all the images obtained were evaluated together (Case 6, Table 3). In a retro- spective analysis done after the end of the study, the stoma in this patient was found to be so narrowed (string shaped) that CSF passing across it was not sufficient to maintain CSF circulation. The flow-void phenomenon seen on 3DSPACE and PC-MR images in this patient was attributed to excessive turbulent flow across narrowed stoma. Endoscopic examination of this patient revealed that the stoma was almost closed, and a repeat ETV was performed.

Scores obtained by evaluating all 3 of the PC-MRI, 3DSPACE, and 3D heavily T2W techniques together (triple combination session) were more accurate and correlated better than the scores obtained in the other sessions $(\mathrm{p}<$ 0.001, Table 4, Fig. 4). No significant difference was found regarding detection of ETV patency among scoring sessions done using PC-MRI, 3D-SPACE, and 3D heavily $\mathrm{T} 2 \mathrm{~W}$ techniques singly, and the 3 techniques correlated 

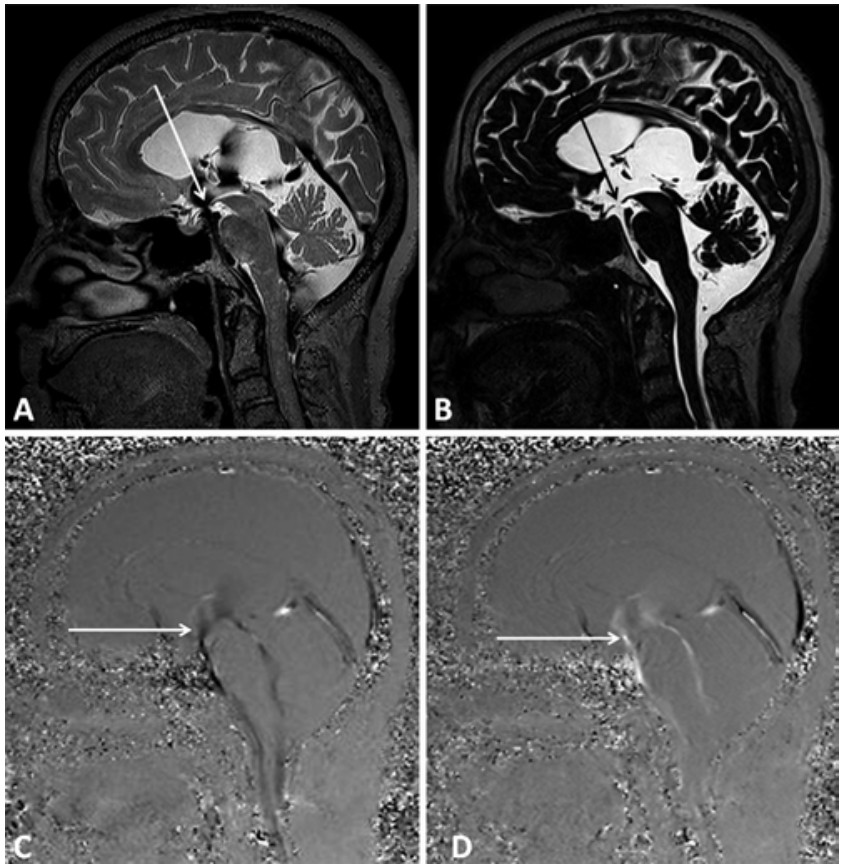

FIG. 3. Prominent CSF flow (flow-void sign) is present in the inferior wall of the third ventricle on a sagittal 3D-SPACE image obtained in a 74-year-old woman with partial aqueductal stenosis and a history of ETV (arrow, A). On the sagittal 3D heavily T2W image, there is a defect in the inferior wall of the third ventricle consistent with patent stoma (arrow, B). Sagittal PC-MR images also demonstrate CSF flow consistent with patent stoma (arrows in C and D). The PC-MRI technique is well matched with 3D-SPACE results.

well with each other $(p<0.001)$. The sensitivity, specificity, negative predictive value, positive predictive value, and accuracy analysis of 3D-SPACE, PC-MRI, and 3D heavily $\mathrm{T} 2 \mathrm{~W}$ techniques are given in Table 5.

\section{Discussion}

Because it is a more physiological method with better outcome relative to the shunt procedures, ETV is being used in increasing frequency in the treatment of obstructive hydrocephalus. ${ }^{7,9}$ Nevertheless, there are no noninvasive, safe, and efficient methods that can be used to follow up with patients after ETV procedures. ${ }^{4,13}$ Although in daily routine practice PC-MRI and 3D heavily T2W sequences are being used together to assess ETV patency,

TABLE 4. Agreement between MR sequences and gold-standard test findings for flow-void phenomenon on PC-MRI and 3D-SPACE techniques, and integrity of third ventricle on 3D heavily T2W images

\begin{tabular}{lll}
\hline \multicolumn{1}{c}{ Scoring Sessions } & Kappa $(95 \% \mathrm{Cl})$ & $\mathrm{p} \mathrm{Value}$ \\
\hline 3D heavily T2W & $0.86(0.45-1.00)$ & $<0.001$ \\
\hline 3D heavily T2W \& PC-MRI & $0.88(0.48-1.00)$ & $<0.001$ \\
\hline 3D heavily T2W \& 3D-SPACE & $0.88(0.49-1.00)$ & $<0.001$ \\
\hline PC-MRI \& 3D-SPACE & $0.88(0.49-1.00)$ & $<0.001$ \\
\hline 3D-SPACE & $0.90(0.51-1.00)$ & $<0.001$ \\
\hline PC-MRI & $0.90(0.51-1.00)$ & $<0.001$ \\
\hline 3D heavily T2W, PC-MRI, \& 3D-SPACE & $1.00(0.62-1.00)$ & $<0.001$ \\
\hline
\end{tabular}
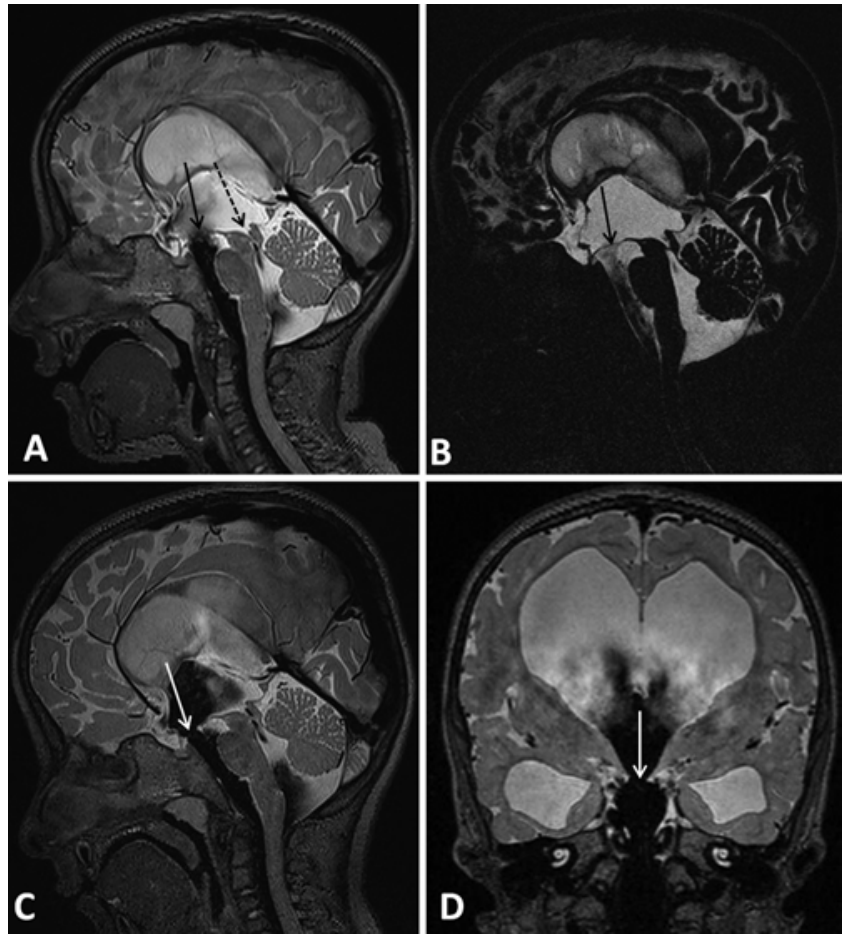

FIG. 4. A 5-year-old girl with a history of ETV underwent MRI because of a complaint of progressive headache. Preoperative sagittal 3DSPACE image shows partial aqueductal stenosis and nonfunctional stoma (broken and solid arrows, respectively; A). On preoperative sagittal 3D heavily $\mathrm{T} 2 \mathrm{~W}$ image the third ventricle inferior wall is intact, consistent with closed stoma (arrow, B). After revision surgery, sagittal and coronal reformatted 3D-SPACE images demonstrate a hypointense flow-void phenomenon consistent with open and functional stoma (arrows in $\mathbf{C}$ and $\mathbf{D}$ ).

these techniques have some limitations. ${ }^{2}$ For instance, PCMRI can be misinterpreted in the presence of turbulent and/or complex flow, or if the velocity encoding value is not correctly chosen. ${ }^{5}$ Besides this, because the PC-MRI technique is $2 \mathrm{D}$ it is sometimes insufficient, or false results may be seen due to partial volume effect. In these situations, PC-MRI acquisition may be required more than once, which lengthens the acquisition time significantly. In addition, to obtain images passing through the level of the stoma, an experienced neuroradiologist is required to evaluate the acquired images simultaneously during scanning for an optimal examination. The PC-MRI technique provides physiological information regarding CSF dynamics.?

TABLE 5. Sensitivity, specificity, negative predictive value, positive predictive value, and accuracy analysis of 3D-SPACE, PC-MRI, and 3D heavily T2W sequences

\begin{tabular}{|c|c|c|c|c|c|}
\hline Sequence & Sensitivity & Specificity & NPV & PPV & Accuracy \\
\hline 3D-SPACE & $100 \%$ & $85 \%$ & $100 \%$ & $95 \%$ & $96 \%$ \\
\hline PC-MRI & $100 \%$ & $85 \%$ & $100 \%$ & $95 \%$ & $96 \%$ \\
\hline 3D heavily T2W & $100 \%$ & $80 \%$ & $100 \%$ & $94 \%$ & $95 \%$ \\
\hline $\begin{array}{l}\text { 3D-SPACE \& PC- } \\
\text { MRI \& 3D } \\
\text { heavily T2W }\end{array}$ & $100 \%$ & $100 \%$ & $100 \%$ & $100 \%$ & $100 \%$ \\
\hline
\end{tabular}

NPV = negative predictive value $; P P V=$ positive predictive value. 
On the other hand, accurate morphological data cannot be obtained with PC-MRI. To achieve that, 3D heavily T2W sequences are added in the MRI protocol most of the time..$^{13}$ However, 3D heavily T2W sequences are not always efficient in imaging the inferior wall of the third ventricle, because it is thinned and/or deformed due to hydrocephalus. ${ }^{2,16}$ Also, turbulent CSF flow passing across the stoma is seen as hypointense on 3D heavily T2W images, which challenges evaluation of stoma morphology. In our study, the technique with which most of the equivocal findings (Score 2) were obtained and which correlated the least with findings of the tests of reference was 3D heavily T2W images (Tables 3 and 4).

Recently, an optimized, single-slab, turbo spin echobased imaging technique with high spatial resolution and signal-to-noise ratio was developed to generate 3D T1, T2, FLAIR, or heavily T2W images with lower specific absorption rate values for 3D head imaging with 3-T machines. ${ }^{11,12}$ With this technique, thinner section images and $3 \mathrm{D}$ data (by using $<1-\mathrm{mm}^{3}$ voxels) can be acquired with acceptable acquisition time (approximately 5 minutes), minimizing the partial volume effect between small entities and surrounding tissue. ${ }^{3,12}$

Our previous studies demonstrated that T2W 3DSPACE with variant flip-angle mode images may show the flow dynamics of CSF in the ventricular system. ${ }^{3,5} \mathrm{Be}-$ sides, it has been shown that 3D data with high resolution acquired with this technique may demonstrate clearly the morphology of the third ventricle. ${ }^{4}$ Findings in this study are consistent with those of previous ones; it is shown that the 3D-SPACE technique could provide the information obtained when 3D heavily T2W and PC-MRI techniques were used together, and most of the patients $(96 \%)$ in the study group were diagnosed correctly by using only the 3D-SPACE technique. Besides, no advantages of PC-MRI and 3D heavily T2W techniques over the 3D-SPACE technique could be shown. These results show that adding the 3D-SPACE sequence to the MRI protocol in the assessment of ETV patency may be sufficient in most patients, without the need for PC-MRI and/or 3D heavily T2W sequences. This approach shortens the total imaging time, increases patient compliance, and decreases cost. On the other hand, evaluation with all 3 of the 3D-SPACE, PCMRI, and 3D heavily T2W images increases diagnostic efficacy up to $100 \%$ (Tables 3-5). This shows that patients with insufficient or equivocal findings on 3D-SPACE images should also be evaluated with the other techniques. The advantages and disadvantages of 3D-SPACE over PCMRI and 3D heavily T2W sequences are given in Table 6.

Although ventriculography is the gold-standard method for the assessment of ETV patency, PC-MRI, due to its noninvasive nature, is preferred and is accepted as a reference method.,16 In this study the findings obtained using the 3D-SPACE technique almost perfectly match the findings of the PC-MRI technique and gold-standard test results (Table 3). The 3D-SPACE or PC-MRI techniques could accurately diagnose all of the patients with closed or nonfunctional stoma, without any false or suspicious results. This shows that the 3D-SPACE technique can efficiently be used alone in the evaluation of patients with ETV.
TABLE 6. Advantages and disadvantages of the 3 sequences

\begin{tabular}{lccc}
\hline & \multicolumn{3}{c}{ Sequences } \\
\cline { 2 - 4 } \multicolumn{1}{c}{ Factor } & $\begin{array}{c}\text { 3D- } \\
\text { SPACE }\end{array}$ & PC-MRI & $\begin{array}{c}\text { 3D Heavily } \\
\text { T2W }\end{array}$ \\
\hline Physiological data & Yes & Yes & No \\
\hline Morphological data & Yes & No & Yes \\
\hline Whole-brain imaging & Yes & No & No \\
\hline Experience requirement & Yes & Yes & No \\
\hline Mean acquisition time in min & $4-5$ & $6-10$ & $4-5$ \\
\hline Comprehensive acquisition & Yes & Yes & Yes \\
\hline $\begin{array}{l}\text { Necessity of ECG or pulse } \\
\text { triggering }\end{array}$ & No & Yes & No \\
\hline 3D acquisition & Yes & No (single slice) & Yes \\
\hline
\end{tabular}

ECG = electrocardiography.

According to Bargalló et al., a prominent or high stroke volume in the stoma appears to be a good indicator of patent ETV and a positive predictor of favorable clinical outcome. ${ }^{6}$ In this study, quantitative PC-MRI parameters in patients with patent ETV were found to be higher than those in patients with closed or nonfunctional stoma (Table 2). These findings show that these parameters are useful in follow-up examinations after an ETV procedure, which is consistent with the previous studies. ${ }^{6}$ It may seem to be a disadvantage that the 3D-SPACE technique does not allow quantitative evaluation, but actually no cutoff value was determined for the use of quantitative parameters in PCMRI studies, and their use in routine practice is limited. In this study as well as in our daily routine practice, no additive value of quantitative evaluation has been shown.

This study in a relatively larger series of patients has some limitations. Most important of these is that not all of the techniques we have used to evaluate ETV stoma could be compared with results of gold-standard methods such as ventriculography or endoscopy in all patients. These comparisons could not be done in all cases because not all patients had undergone endoscopy, and ventriculography is an invasive method. The second most important limitation of the study is that analyses of inter/intraobserver agreement and of confidence in radiological reporting were not done for the 3D-SPACE, PC-MRI, and 3D heavily $\mathrm{T} 2 \mathrm{~W}$ techniques. On the other hand, in a previous study we performed in patients with suspected spontaneous third ventriculostomy in which we evaluated the integrity of the third ventricle, we concluded that these values were higher with the 3D-SPACE technique. ${ }^{5}$ Therefore a reliability analysis was not obtained for this study.

\section{Conclusions}

The 3D-SPACE technique with variant flip-angle mode provides morphological and physiological data rapidly and efficiently. We suggest the use of 3D-SPACE as a standalone, first-line sequence in addition to routine brain MRI protocols in assessing patients with ETV, thereby decreasing scan time, and reserving the use of a combination of additional sequences such as PC-MRI and 3D heavily $\mathrm{T} 2 \mathrm{~W}$ images in suspicious or complex cases. 


\section{Acknowledgments}

We gratefully acknowledge M. Sahin Ugurel, MD (Professor of Neuroradiology), M. Gulbiz Kartal, MD, and Musa Kurnaz (our experienced technician) for their excellent contributions.

\section{References}

1. Algin O: Radiologic evaluation of spontaneous or endoscopic third ventriculostomy: which technique is more useful? Clin Neurol Neurosurg 113:700-702, 2011

2. Algin O, Hakyemez B, Parlak M: Phase-contrast MRI and 3D-CISS versus contrast-enhanced MR cisternography for the detection of spontaneous third ventriculostomy. J Neuroradiol 38:98-104, 2011

3. Algin O, Turkbey B: Evaluation of aqueductal stenosis by 3D sampling perfection with application-optimized contrasts using different flip angle evolutions sequence: preliminary results with 3T MR imaging. AJNR Am J Neuroradiol 33:740-746, 2012

4. Algin O, Turkbey B: Intrathecal gadolinium-enhanced MR cisternography: a comprehensive review. AJNR Am J Neuroradiol 34:14-22, 2013

5. Algin O, Turkbey B, Ozmen E, Ocakoglu G, Karaoglanoglu M, Arslan H: Evaluation of spontaneous third ventriculostomy by three-dimensional sampling perfection with application-optimized contrasts using different flip-angle evolutions (3D-SPACE) sequence by 3T MR imaging: preliminary results with variant flip-angle mode. J Neuroradiol 40:11-18, 2013

6. Bargalló N, Olondo L, Garcia AI, Capurro S, Caral L, Rumia $\mathrm{J}$ : Functional analysis of third ventriculostomy patency by quantification of CSF stroke volume by using cine phase-contrast MR imaging. AJNR Am J Neuroradiol 26:2514-2521, 2005

7. Bilginer B, Oguz KK, Akalan N: Endoscopic third ventriculostomy for malfunction in previously shunted infants. Childs Nerv Syst 25:683-688, 2009

8. Di X, Ragab M, Luciano MG: Cine phase-contrast MR images failed to predict clinical outcome following ETV. Can J Neurol Sci 36:643-647, 2009

9. Hellwig D, Grotenhuis JA, Tirakotai W, Riegel T, Schulte DM, Bauer BL, et al: Endoscopic third ventriculostomy for obstructive hydrocephalus. Neurosurg Rev 28:1-38, 2005

10. Hodel J, Decq P, Rahmouni A, Bastuji-Garin S, Maraval A,
Combes C, et al: Brain ventricular wall movement assessed by a gated cine MR trueFISP sequence in patients treated with endoscopic third ventriculostomy. Eur Radiol 19:27892797, 2009

11. Hodel J, Lebret A, Petit E, Leclerc X, Zins M, Vignaud A, et al: Imaging of the entire cerebrospinal fluid volume with a multistation 3D SPACE MR sequence: feasibility study in patients with hydrocephalus. Eur Radiol 23:1450-1458, 2013

12. Kartal MG, Algin O: Evaluation of hydrocephalus and other cerebrospinal fluid disorders with MRI: an update. Insights Imaging 5:531-541, 2014

13. Kunz M, Schulte-Altedorneburg G, Uhl E, Schmid-Elsaesser R, Schöller K, Zausinger S: Three-dimensional constructive interference in steady-state magnetic resonance imaging in obstructive hydrocephalus: relevance for endoscopic third ventriculostomy and clinical results. J Neurosurg 109:931938, 2008

14. Mugler JP III, Bao S, Mulkern RV, Guttmann CR, Robertson RL, Jolesz FA, et al: Optimized single-slab three-dimensional spin-echo MR imaging of the brain. Radiology 216:891899,2000

15. Mugler JP III, Bauer S, Paul D, Stemmer A, Kiefer B: 2368. Radial single-slab 3D turbo spin echo (SPACE). Presented at the International Society for Magnetic Resonance in Medicine 21st Annual Meeting \& Exhibition, Salt Lake City, Utah, 2013 (Poster) (http://www.ismrm.org/13/tp05.htm) [Accessed February 25, 2015]

16. Pople IK: Hydrocephalus and shunts: what the neurologist should know. J Neurol Neurosurg Psychiatry 73 (Suppl 1): i17-i22, 2002

\section{Author Contributions}

Conception and design: Ucar, Borcek. Acquisition of data: Algin, Ucar, Borcek. Analysis and interpretation of data: Ozmen. Critically revising the article: Tali, Ozisik. Statistical analysis: Ocakoglu. Study supervision: Ozisik.

\section{Correspondence}

Oktay Algin, Department of Radiology, Ataturk Education and Research Hospital, Bilkent, Ankara 06000, Turkey. email: droktayalgin@gmail.com. 